\title{
The Sri Lankan paradox: high genetic diversity in Plasmodium vivax populations despite decreasing levels of malaria transmission - ERRATUM
}

\author{
SHARMINI GUNAWARDENA, MARCELO U. FERREIRA, G. M. G. KAPILANANDA, \\ DYANN F. WIRTH and NADIRA D. KARUNAWEERA
}

doi:10.1017/S0031182013002278, published online by Cambridge University Press 14 February 2014.

In the above recently published article, the last line in Table 1 was mistakenly misaligned. The correct version of the Table appears below. The publisher apologises for this error.

Table 1. Characterization of the 14 polymorphic $P$. vivax microsatellite loci $(n=185)$

\begin{tabular}{|c|c|c|c|c|c|c|c|}
\hline \multirow[b]{2}{*}{ Locus } & \multirow{2}{*}{$\begin{array}{l}\text { Allele size } \\
\text { range }(r)\end{array}$} & \multirow{2}{*}{$\begin{array}{l}\text { Number of } \\
\text { alleles (k) }\end{array}$} & \multirow[b]{2}{*}{$H_{E}$} & \multirow{2}{*}{$\begin{array}{l}\text { M ratio } \\
(\mathrm{k} / \mathrm{r})\end{array}$} & \multicolumn{3}{|c|}{ Temporal estimation of $\mathrm{M}$ ratio } \\
\hline & & & & & $2003-4$ & 2005 & $2006-7$ \\
\hline MS1 & $201-249(48)$ & 16 & $0 \cdot 8998$ & $0 \cdot 333$ & $0 \cdot 333$ & $0 \cdot 289$ & $0 \cdot 289$ \\
\hline MS2 & $174-286(112)$ & 18 & $0 \cdot 8263$ & $0 \cdot 161$ & $0 \cdot 231$ & $0 \cdot 196$ & $0 \cdot 135$ \\
\hline MS3 & $152-197(45)$ & 15 & $0 \cdot 8706$ & $0 \cdot 333$ & $0 \cdot 289$ & $0 \cdot 311$ & $0 \cdot 214$ \\
\hline MS4 & $177-222(45)$ & 12 & $0 \cdot 8015$ & $0 \cdot 267$ & $0 \cdot 256$ & $0 \cdot 278$ & $0 \cdot 333$ \\
\hline MS5 & $139-232(93)$ & 19 & $0 \cdot 8282$ & $0 \cdot 204$ & $0 \cdot 151$ & $0 \cdot 126$ & $0 \cdot 188$ \\
\hline MS6 & $201-273(72)$ & 22 & $0 \cdot 9145$ & $0 \cdot 306$ & $0 \cdot 238$ & $0 \cdot 236$ & $0 \cdot 136$ \\
\hline MS7 & $127-178(51)$ & 17 & $0 \cdot 8301$ & $0 \cdot 333$ & $0 \cdot 256$ & $0 \cdot 333$ & $0 \cdot 176$ \\
\hline MS8 & $165-345(180)$ & 46 & $0 \cdot 9657$ & $0 \cdot 256$ & $0 \cdot 175$ & $0 \cdot 183$ & $0 \cdot 150$ \\
\hline MS9 & $140-224(84)$ & 16 & $0 \cdot 8507$ & $0 \cdot 190$ & $0 \cdot 160$ & $0 \cdot 267$ & $0 \cdot 178$ \\
\hline MS10 & $177-291(114)$ & 20 & $0 \cdot 9155$ & $0 \cdot 175$ & $0 \cdot 222$ & $0 \cdot 167$ & $0 \cdot 175$ \\
\hline MS12 & $194-260(66)$ & 21 & 0.9225 & $0 \cdot 318$ & $0 \cdot 270$ & $0 \cdot 254$ & $0 \cdot 242$ \\
\hline MS15 & $236-293(57)$ & 14 & $0 \cdot 8637$ & $0 \cdot 246$ & $0 \cdot 196$ & $0 \cdot 196$ & $0 \cdot 375$ \\
\hline MS16 & $138-375(237)$ & 35 & $0 \cdot 8153$ & $0 \cdot 148$ & $0 \cdot 089$ & $0 \cdot 105$ & $0 \cdot 027$ \\
\hline \multirow[t]{2}{*}{ MS20 } & $150-246(96)$ & 31 & 0.9369 & $0 \cdot 323$ & $0 \cdot 278$ & $0 \cdot 260$ & $0 \cdot 107$ \\
\hline & Mean & $21 \cdot 57$ & $0 \cdot 8744$ & $0 \cdot 2570$ & & & \\
\hline
\end{tabular}

REFERENCE

Gunawardena, S., Ferreira, M. U., Kapilananda, G. M. G., Wirth, D. F. and Karunaweera, N. D. The Sri Lankan paradox: high genetic diversity in Plasmodium vivax populations despite decreasing levels of malaria transmission. Parasitology, published online 14 February 2014, doi:10.1017/ S0031182013002278. 